\title{
Fear Conditioning, Safety Learning, and Sleep in Humans
}

\author{
Anisa J. Marshall, ${ }^{1}$ Dean T. Acheson, ${ }^{1,3,4}$ Victoria B. Risbrough, ${ }^{1,3,4}$ Laura D. Straus, ${ }^{1,5}$ and Sean P.A. Drummond ${ }^{2,3,4,5}$ \\ ${ }^{1}$ Research and ${ }^{2}$ Psychology Services, Veterans Affairs San Diego Healthcare System, San Diego, California 92161, ${ }^{3}$ Veterans Affairs San Diego Center for \\ Excellence in Stress and Mental Health, San Diego, California 92161, ${ }^{4}$ Department of Psychiatry, University of California San Diego, San Diego, California \\ 92093-0603, and 5SDSU-UCSD Joint Doctoral Program in Clinical Psychology; San Diego, California 92120
}

Fear conditioning is considered an animal model of post-traumatic stress disorder. Such models have shown fear conditioning disrupts subsequent rapid eye movement sleep (REM). Here, we provide a translation of these models into humans. Using the fear potentiated startle (FPS) procedure, we examined the effects of fear conditioning and safety signal learning on subsequent REM sleep in healthy adults. We also examined the effects of changes in REM sleep on retention of fear and safety learning. Participants $(n=42$ normal controls) spent 3 consecutive nights in the laboratory. The first was an adaptation night. Following the second night, we administered a FPS procedure that included pairing a wrist shock with a threat signal and a safety signal never paired with a shock. The next day, we administered the FPS procedure again, with no wrist shocks to any stimulus, to measure retention of fear and safety. Canonical correlations assessed the relationship between FPS response and REM sleep. Results demonstrated that increased safety signal learning during the initial acquisition phase was associated with increased REM sleep consolidation that night, with $28.4 \%$ of the variance in increased REM sleep consolidation from baseline accounted for by safety signal learning. Overnight REM sleep was, in turn, related to overnight retention of fear and safety learning, with $22.5 \%$ of the variance in startle retention accounted for by REM sleep. These data suggest that sleep difficulties, specifically REM sleep fragmentation, may play a mechanistic role in post-traumatic stress disorder via an influence on safety signal learning and/or threat-safety discrimination.

Key words: fear conditioning; REM sleep; safety learning; translational

\section{Introduction}

Sleep difficulties are among the most common symptoms in post-traumatic stress disorder (PTSD) (Germain et al., 2008; Spoormaker and Montgomery, 2008), with 70\% of civilians (Ohayon and Shapiro, 2000), and up to $90 \%$ of combat veterans with PTSD (Neylan et al., 1998) reporting sleep difficulties. More than just a symptom, sleep appears to play a role in development and maintenance of PTSD (Germain et al., 2008). Although sleep disruption has been linked with the development, exacerbation, and possibly poor recovery from PTSD (Babson and Feldner, 2010; Nadorff et al., 2011; van Liempt et al., 2013), the exact mechanism underlying the role sleep plays in PTSD is not well known.

PTSD is a disorder of abnormal fear processes, wherein learned fear responses are greater and more difficult to inhibit (Johnson et al., 2012). One translational model for addressing the

Received Feb. 3, 2014; revised July 15, 2014; accepted July 18, 2014.

Author contributions: D.T.A., V.B.R., and S.P.A.D. designed research; A.J.M., D.T.A., L.D.S., and S.P.A.D. performed research; A.J.M., D.T.A., V.B.R., L.D.S., and S.P.A.D. analyzed data; A.J.M., D.T.A., V.B.R., L.D.S., and S.P.A.D. wrote the paper.

This work was supported by Defense Medical Research and Development Program DM102425. Infrastructure support was provided by Veterans Administration San Diego Center for Excellence in Stress and Mental Health.

The authors declare no competing financial interests.

Correspondence should be addressed to Dr. Sean P.A. Drummond, Veterans Administration San Diego Healthcare System, MC 116B, 3350 La Jolla Village Drive, San Diego, CA 92161. E-mail: drummond@ucsd.edu.

A.J. Marshall's current address: Stanford University School of Medicine, Department of Neurology \& Neurological Sciences.

DOI:10.1523/JNEUROSCI.0478-14.2014

Copyright $\odot 2014$ the authors $\quad 0270-6474 / 14 / 3411754-07 \$ 15.00 / 0$ role of sleep in fear processes, and thus PTSD, is fear conditioning. Fear conditioning is a Pavlovian response whereby a neutral stimulus is paired with an aversive stimulus until the previously neutral stimulus elicits a conditioned fear response and is considered a good animal model of PTSD (Grillon, 2002). Animal models have shown consistent relationships between fear conditioning and sleep, especially rapid eye movement (REM) sleep. For example, fear conditioning disrupts and fragments REM sleep (Sanford et al., 2001; Wellman et al., 2008), even after a single trial of conditioning (Sanford et al., 2003). REM sleep fragmentation or deprivation, in turn, impairs extinction memory, the process by which an animal learns the previously feared stimulus no longer signals threat (Silvestri, 2005; Fu et al., 2007). Furthermore, greater REM sleep fragmentation before fear conditioning predicts greater acoustic startle responses 1 month after fear conditioning (Polta et al., 2013). These findings suggest that REM sleep disruption may play a role in PTSD via interactions with fear and/or extinction processes. Few studies, though, have translated the animal model findings into humans. While studies have shown sleep in general (Pace-Schott et al., 2009; Sturm et al., 2013), and REM sleep in particular (Spoormaker et al., 2010, 2012), play a role in extinction memory in humans, no published studies have examined whether initial fear conditioning disrupts overnight REM sleep in humans.

In contrast to a conditioned threat signal, fear conditioning procedures can also include a safety signal. This is a stimulus presented during fear learning but never paired with the aversive unconditioned stimulus (US). Impairments in safety signal learning are seen when someone shows a strong fear response to 


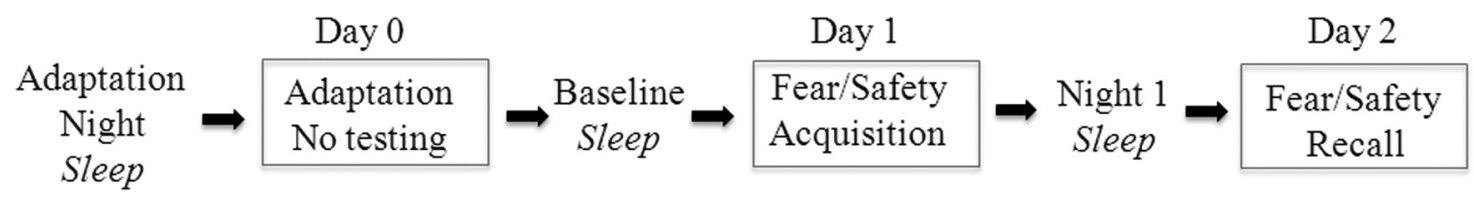

Figure 1. Study time line. Participants entered the laboratory on the evening before the Adaptation Night and remained there until the study was completed. Startle procedure was scheduled for $10-12 \mathrm{~h}$ after awakening.

\section{Day $1 \quad$ Day 2}

Acquisition Phase

Habituation: 6 NA Presentations

Acquisition: 4 CS Blocks

Prototypical Block:

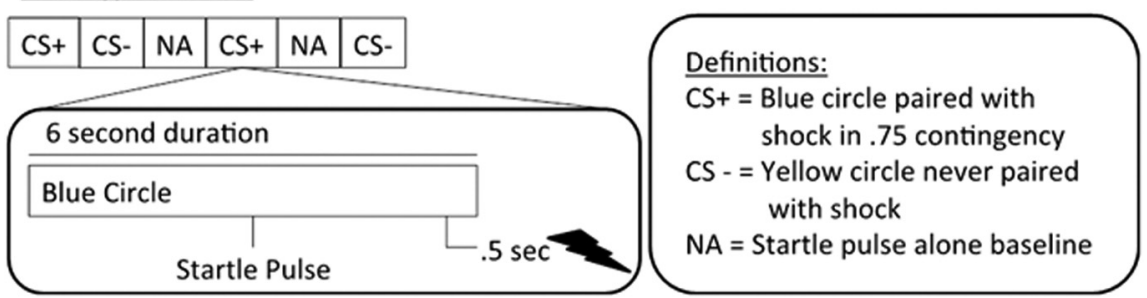

Figure 2. Fear potentiated startle procedure. Schematic of the fear potentiated startle procedure detailing the fear acquisition and recall phases, as well as illustrating a prototypical block and trial.

a safety signal (e.g., as strong as that shown to the threat signal), despite repeated presentations of the stimulus without ever pairing it with the aversive stimulus. There are no published studies examining sleep in relation to safety signals. However, given PTSD is the only clinical population to show impairments in safety signal learning (Jovanovic et al., 2010), it is of interest to know if safety learning interacts with REM sleep, as well.

Our aim was to examine effects of fear conditioning and safety learning on subsequent sleep in humans. Our main hypothesis, based on the animal literature, was greater levels of fear conditioning during the day would lead to more fragmented REM sleep at night. Although there is no literature upon which to develop hypotheses related to safety learning, we anticipated the opposite relationship: greater levels of safety learning would be associated with consolidated REM sleep. We also examined whether changes in REM sleep following fear/safety learning was associated with next day retention of conditioned responses.

\section{Materials and Methods}

Participants. Forty-two healthy normal controls were recruited from the San Diego area, completed the study, and were included in these analyses (age $=24.1 \pm 5.0$ years, $42 \%$ female, $37 \%$ racial/ethnic minority). In addition, one subject was excluded because of invalid sleep data, and 13 were excluded for not showing a startle response to 12108 - $\mathrm{dB}$ acoustic pulses administered at screening (startle reactivity was defined as exhibiting responses to $>50 \%$ of trials). After written informed consent, participants were screened for drug use, sleep disorders, and psychiatric and medical conditions via structured interview and laboratory tests. Inclusion criteria included the following: (1) age 18-39 years old; (2) $\geq 12$ years of education; and (3) consistent sleep-wake schedule, including 7-9 h of overnight sleep/night. Female participants were studied in the early follicular phase of the menstrual cycle, as estrogen can affect fear learning processes (Milad et al., 2006). Participants maintained a regular sleep-wake schedule, corresponding to their habitual schedule, for 1 week before entering the laboratory portion of the study. Sleep diaries and actigraphy monitored adherence with this schedule, and anyone deviating $>15$ min on $\geq 2$ nights was not studied. Participants refrained from alcohol and caffeine for $48 \mathrm{~h}$ before entering the laboratory.

Sleep procedures. Participants entered the laboratory $\sim 2 \mathrm{~h}$ before bed time on the adaptation night and lived in the laboratory until the end of the study, including three overnight sleep studies (Fig. 1). Each night, a polysomnogram, including EEG, EOG, and chin EMG, monitored sleep. To screen for unreported sleep apnea and periodic leg movements, monitors were added on adaptation night. Three REM sleep variables served as our main measures: REM\% (proportion of sleep spent in REM); REM sleep efficiency (RE: proportion of epochs within REM episodes scored as REM sleep, as opposed to wake or non-REM sleep; this is parallel to the concept of sleep efficiency); and REM sleep latency (RL: time from sleep onset to the first REM epoch). We took lower REM\%, lower RE, and shorter RL as indices of REM sleep fragmentation. To examine change in REM sleep after the startle procedure, a difference score was calculated as follows: (Night 1 - baseline).

Fear potentiated startle (FPS). We used FPS model of cued fear conditioning. Participants learned to associate a visual conditioned stimulus (CS) with an US of electrical shock to the wrist. Conditioned fear responses to the CS were measured using eyeblink magnitude in response to acoustic stimuli presented in the presence/absence of CS. The degree of relative startle potentiation in the presence of the CS, compared with absence of CS, was used as an operational measure of fear response.

On Day 1, participants completed the fear-conditioning procedure to acquire conditioned FPS, as described in detail previously (Acheson et al., 2013). Participants were seated in a lounge chair in a soundattenuated testing chamber. The visual CSs (colored circles) were presented using an LCD computer monitor connected to a Dell desktop computer. Startle pulses were $108 \mathrm{~dB}, 40 \mathrm{~ms}$ bursts of broadband noise. EMG responses were recorded from electrodes placed at the orbicularis oculi muscle (San Diego Instruments; for details on apparatus, startle measurement and processing see Acheson et al., 2013). The electrical shock stimuli were delivered via a Contact Precision Instruments SHK1 aversive shock stimulator coupled with an IBM ThinkPad notebook computer as previously described by Acheson et al. (2013). On the first day of testing, shock intensity levels were set manually for each individual by delivering gradually more intense shocks $(0-5 \mathrm{~mA}$ range) until the subject reported that the shock level was "highly annoying yet not painful." Figure 2 contains a schematic of the FPS procedure. Acquisition began with a $1 \mathrm{~min}$ acclimation period consisting of $70 \mathrm{db}$ of white noise followed by six startle pulses presented in the absence of any other stimuli in order for the participants to acclimate/habituate startle responses to baseline level. After acclimation, visual cues were as follows: (1) conditioned via pairing with wrist shock in 0.75 contingency $\left(\mathrm{CS}^{+}\right)$; or (2) never paired with the shock $\left(\mathrm{CS}^{-}\right)$, thus serving as safety signals. The acquisition session included $8 \mathrm{CS}^{+}$trials (6 paired with shock), $8 \mathrm{CS}^{-}$ trials, and 8 noise alone (NA) trials where no visual stimuli were presented, providing baseline startle reactivity. The auditory stimulus during the NA trials, a brief $(40 \mathrm{~ms})$ pulse of $108 \mathrm{~dB}$ is used to induce a startle response, the magnitude of which is the operational measure of threat response or "fear." The auditory stimulus is not a unconditioned 
stimulus, as it is presented during all conditions (NA, $\mathrm{CS}^{-}$, and $\mathrm{CS}^{+}$ trials). Thus, because this probe happens in every trial type, it is not predicted by the presence or absence of any specific stimulus. To the extent the startle pulse has arousing/aversive qualities, then such an effect is controlled for by using a difference score of potentiation above background for the stimuli of interest (i.e., the differential between NA trials and the $\mathrm{CS}^{+}$or $\mathrm{CS}^{-}$trials). Stimulus presentation was block randomized with the constraint of two trials of each type $\left(\mathrm{CS}^{+}, \mathrm{CS}^{-}\right.$, and NA) per block. This approach prevents confounds of uneven habituation effects on any one stimulus type and assures accurate temporal match of $\mathrm{NA}$ baseline responses to $\mathrm{CS}^{+}$and $\mathrm{CS}^{-}$trials. Participants returned on Day 2 for the recall phase. FPS recall was assessed via four presentations of each stimulus type ( $\mathrm{CS}^{+}, \mathrm{CS}^{-}$, and NA) in block randomized order as in acquisition. No shocks were presented during this phase.

Data were analyzed by averaging peak responses to each stimulus type within a block. Responses were examined trial by trial to remove voluntary eyeblink and movement artifact, with responses only being scored if they were within $100 \mathrm{~ms}$ of the onset of the startle pulse. The NA average was subtracted from the $\mathrm{CS}^{+}$and $\mathrm{CS}^{-}$responses to create a score representing startle above baseline for each CS type in each block (e.g., $\left(\mathrm{CS}^{+}\right)$- (NA)) (Norrholm et al., 2006, 2011; Acheson et al., 2013). Three variables served as outcomes for the acquisition phase: (1) acquisition to the threat signal (i.e., fear potentiation): mean $\mathrm{CS}^{+}$response during the last two blocks of the session; (2) safety signal learning: difference in $\mathrm{CS}^{-}$response from the first block to the last block; and (3) differential conditioning: difference between mean $\mathrm{CS}^{+}$versus $\mathrm{CS}^{-}$responses during last two blocks (i.e., differentiation of threat from safety signals). Safety signal learning is measured slightly differently than acquisition to the threat signal and differential conditioning because it is conceptualized as a within-subject change across the session (Norrholm et al., 2011; Acheson et al., 2013). The extent to which a subject learns over time that the $\mathrm{CS}^{-}$is, indeed, a safety signal, is best captured by examining the reduction in potentiation to the $\mathrm{CS}^{-}$stimulus from the beginning of the task (when they do not know it represents safety) to the end of the task (when they should have learned it is a safety signal). Variables of interest on Day 2 related to retention of learning from Day 1: (1) fear retention: change in response to $\mathrm{CS}^{+}$from the last block of Day 1 to the first block of Day 2; (2) safety signal retention: change in response to $\mathrm{CS}^{-}$from the last block of Day 1 to the first block of Day 2; and (3) retention of differential learning: difference in response to $\mathrm{CS}^{+}$and $\mathrm{CS}^{-}$during first two blocks of Day 2.

Statistical analyses. To document the anticipated differential effects between fear potentiation to the $\mathrm{CS}^{+}$and safety signal learning on the $\mathrm{CS}^{-}$, we compared the FPS response to each stimulus type during the relevant blocks, as defined above, with a paired-samples $t$ test.

For the main analyses, because we anticipated two startle variables from Day 1 (fear acquisition and safety signal learning) being associated with three REM sleep variables (RE, REM\% RL), we used a multivariate approach: canonical correlation analysis. This analysis forms canonical variates (i.e., latent variables) from each set of input variables, assessing the correlation between those canonical variates. If that correlation is significant, one examines the correlation (i.e., structural coefficients) between individual measures and their respective canonical variate to determine which measures make significant contributions to the overall relationship (Thompson, 2000; Tabachnick and Fidell, 2001). Based on recommendations of Tabachnick and Fidell (2001), we considered any structural coefficient $>|0.30|$ to indicate a significant contribution to the respective canonical variate and thus the overall relationship between FPS and REM sleep measures.

After determining the relationship between fear/safety acquisition and subsequent REM sleep, we examined whether those changes in REM sleep on Night 1 were associated with overnight retention of fear/safety, as measured on Day 2. Here, we used a similar multivariate approach using the REM canonical variate developed in the analysis above to predict response to FPS measures on Day 2. Outcome variables were as follows: fear retention, safety retention, and differential conditioning retention. For this analysis, those participants who did not show a potentiation response to the threat signal on Day 1 (potentiation response defined as $\left.\left(\mathrm{CS}^{+}\right)-(\mathrm{NA})>0\right)$ were dropped because, in those individ- uals, our operational measure of fear responding did not exist and thus there was no fear conditioning to "retain" on Day 2. The reason for this is that some people require very loud stimuli $(>108 \mathrm{~dB})$ to show a reliable startle response, or they habituate their startle response very quickly. Subjects who do not startle to the $108 \mathrm{db}$ stimulus provide no data with which we could assess fear conditioning and safety signal learning. Additionally, it is unclear whether a small startle response to the safety signal in those participants was due to learning safety or simply an overall nonresponse to the task. Similar exclusions for low baseline response rates also occur in studies using other physiological measures, such as galvanic skin response. Given this exclusion, the sample size for this analysis was 38. As with the first analysis, significance testing with $\alpha=$ 0.05 was used to determine whether REM sleep predicted the FPS measures and structural coefficients $>|0.30|$ defined which measures made significant contributions to the startle retention canonical variate.

After determining the findings from the main analyses above, two post hoc analyses were conducted to examine alternative explanations for the relationships between FPS and REM sleep reported here. First, we conducted a canonical correlation to determine whether initial REM sleep adaptation to the sleep laboratory predicted fear and/or safety acquisition. Specifically, we calculated change in REM sleep variables across the initial nights as (baseline - adaptation). We then entered those variables into the canonical correlation as one set and the same FPS variables used in the main analysis (i.e., fear potentiation and safety learning) as the other set. Second, to test whether REM sleep contributes variance to startle retention above and beyond that accounted for by initial startle acquisition, we conducted another canonical correlation, including both the startle and REM sleep canonical variates as predictors of startle retention. Here, we entered both startle canonical variate and REM sleep canonical variate as predictors of our three startle retention variables into a canonical correlation. For both post hoc analyses, we evaluated the outcome in the same manner described above.

Examination of age and sex showed neither one was related to any sleep measure, FPS variable, or canonical variate. Thus, they were not included in the analyses.

\section{Results}

Table 1 shows sleep measures from adaptation, baseline, and Night 1. We observed the expected improvements in sleep from adaptation to baseline, and no sleep variable showed further significant changes between baseline and Night 1 within the entire sample. Figure 3 demonstrates the expected differential response to $\mathrm{CS}^{+}$and $\mathrm{CS}^{-}$at the end of the acquisition phase. There was no difference in the response to $\mathrm{CS}^{+}$and $\mathrm{CS}^{-}$at Block $1\left(t_{(41)}=\right.$ $-0.502, p=0.618)$, whereas there was a significant difference at the end of acquisition $\left(t_{(41)}=-2.44, p=0.019\right)$.

Figure $4 A$ shows results of the canonical correlation testing whether FPS measures on Day 1 were associated with changes in REM sleep on Night 1 . The two sets of measures shared one significant factor (Hotelling's Trace $F_{(6,72)}=2.44, p=0.033, r^{2}=$ 0.284 ). Only safety signal learning contributed in a meaningful way to the startle acquisition canonical variate. Standardized canonical coefficients (equivalent to standardized $\beta$ weights in a regression analysis) were as follows: safety signal learning $=1.00$, fear acquisition $=-0.116$, confirming the findings from the structural coefficients. All three REM sleep variables made meaningful contributions to the REM sleep canonical variate. Standardized canonical coefficients were as follows: $\mathrm{RE}=0.466$; REM $\%=0.652 ; \mathrm{RL}=0.589$. The direction of the relationships between the REM sleep measures and the REM canonical variate were all in the direction of more consolidated REM sleep.

Figure $4 B$ shows results of the analysis assessing the relationship between REM sleep and retention of fear/safety on Day 2 . REM sleep significantly predicted values on the retention canonical variate (Hotelling's Trace $F_{(3,34)}=3.29, p=0.032, r^{2}=0.225$ ). Only differential conditioning retention made a meaningful contribution 
Table 1. Sleep measures ${ }^{a}$

\begin{tabular}{|c|c|c|c|c|c|c|c|}
\hline \multirow[b]{2}{*}{ Measure } & \multirow[b]{2}{*}{ Adaptation (mean \pm SD) } & \multirow[b]{2}{*}{ Baseline (mean $\pm S D$ ) } & \multirow[b]{2}{*}{ Night 1 (mean \pm SD) } & \multicolumn{2}{|c|}{ Adaptation - baseline } & \multicolumn{2}{|c|}{ Baseline - Night 1} \\
\hline & & & & $p$ & Effect size & $p$ & Effect size \\
\hline Total sleep Time (min) & $438.6 \pm 45.9$ & $463.0 \pm 43.7$ & $467.6 \pm 35.2$ & $\begin{array}{l}0.00 \\
0.00\end{array}$ & $\begin{array}{r}-0.57 \\
0.58\end{array}$ & 0.32 & -0.16 \\
\hline Wake after sleep onset (min) & $48.6 \pm 34.0$ & $30.1 \pm 21.6$ & $28.1 \pm 17.0$ & & & 0.49 & 0.11 \\
\hline Sleep latency (min) & $12.8 \pm 11.1$ & $13.5 \pm 14.7$ & $10.8 \pm 8.4$ & $\begin{array}{l}0.78 \\
0.01\end{array}$ & $\begin{array}{l}-0.04 \\
-0.44\end{array}$ & 0.21 & 0.22 \\
\hline Sleep efficiency (TST/TIB) & $87.8 \pm 7.4$ & $91.4 \pm 6.0$ & $92.3 \pm 3.9$ & & & 0.28 & -0.18 \\
\hline N1\% & $8.6 \pm 4.7$ & $7.5 \pm 3.3$ & $7.9 \pm 2.9$ & 0.16 & 0.23 & 0.37 & -0.14 \\
\hline $\mathrm{N} 2 \%$ & $53.6 \pm 6.6$ & $49.8 \pm 6.8$ & $48.4 \pm 6.5$ & 0.00 & 0.67 & 0.08 & 0.28 \\
\hline $\mathrm{N} 3 \%$ & $17.9 \pm 8.1$ & $18.9 \pm 7.3$ & $19.0 \pm 6.9$ & $\begin{array}{l}0.15 \\
0.00\end{array}$ & $\begin{array}{l}-0.23 \\
-0.74\end{array}$ & 0.83 & -0.03 \\
\hline REM \% & $20.0 \pm 5.4$ & $23.9 \pm 5.0$ & $24.6 \pm 4.3$ & 0.00 & 0.60 & 0.26 & -0.18 \\
\hline REM latency (min) & $106.9 \pm 56.9$ & $77.4 \pm 22.6$ & $76.8 \pm 26.9$ & & & 0.88 & 0.02 \\
\hline REM efficiency (\%) & $89.4 \pm 7.5$ & $87.9 \pm 8.1$ & $87.1 \pm 9.2$ & 0.32 & 0.16 & 0.57 & 0.09 \\
\hline
\end{tabular}

${ }^{a}$ Sleep measures on adaptation night, baseline, and Night 1. $p$ values are for paired sample $t$ test comparing each night. Effect size is Cohen's $d$.

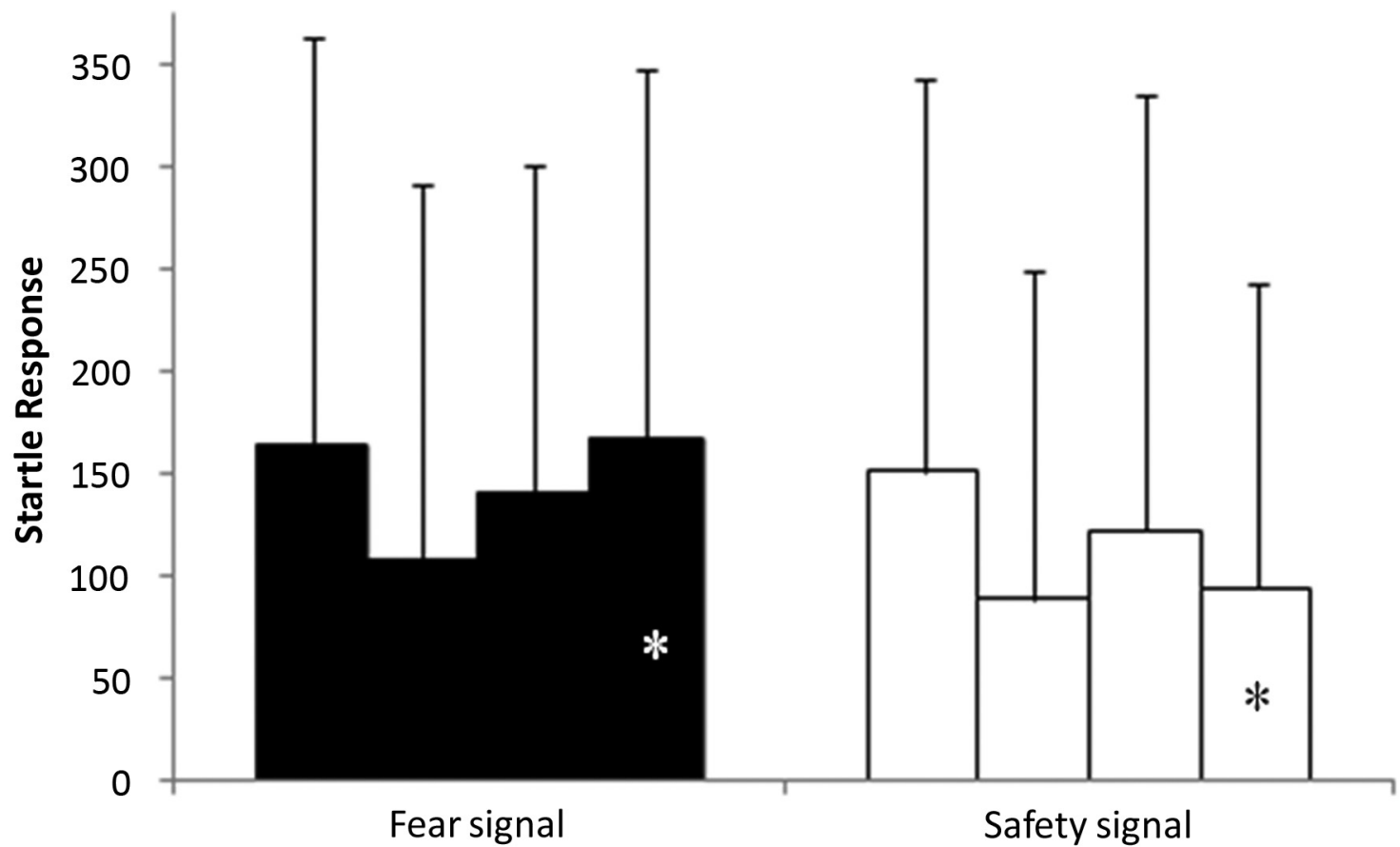

Figure 3. Startle acquisition. Startle response to fear/threat signal (left) and safety signal (right) across 4 blocks of initial acquisition phase. ${ }^{*}$ Significant difference at the end of acquisition between startle response to threat signal and safety signal $(p<0.05)$. Data are mean \pm SD.

to the canonical variate (which we labeled startle retention). Standardized canonical coefficients were as follows: fear retention $=$ 0.322 , safety retention $=0.303$, differential conditioning retention $=1.07$, confirming that differential conditioning retention was the startle retention measure most strongly influenced by REM sleep.

The first post hoc analysis showed there is no association between the extent of habituation in REM sleep to the sleep lab and initial startle acquisition (Hotelling's Trace $F_{(6,72)}=0.372, p=$ $\left.0.985, r^{2}=0.057\right)$. The second post hoc analysis, assessing the combined contribution of startle acquisition and REM sleep to startle retention, was significant (Hotelling's Trace $F_{(6,64)}=2.61$, $\left.p=0.025, r^{2}=0.286\right)$. Both the startle and REM sleep canonical variates made meaningful contributions to the relationship, with very similar correlations to the new predictor canonical variate $(r=0.912$ and 0.837 , respectively). This was confirmed by the structural coefficients (startle latent $=0.649$; REM latent $=$
0.487). With respect to the startle retention variables, the basic results did not change, and the structural coefficients confirmed differential conditioning was the measure most strongly affected by the combination of the initial startle response and REM sleep (standardized canonical coefficients: fear retention $=-0.011$; safety retention $=0.036$, differential conditioning retention $=$ 0.996).

\section{Discussion}

This study examined the relationship between strength of FPS (fear conditioning and safety signal learning) and REM sleep in healthy humans. Our findings did not directly replicate findings in animals, where fear conditioning leads to fragmentation and disruption of REM sleep. Rather, we found that subsequent sleep was more robustly influenced by the extent of safety signal learning. Specifically, stronger safety signal learning was associated with increased REM sleep consolidation that night, with $28.4 \%$ of 

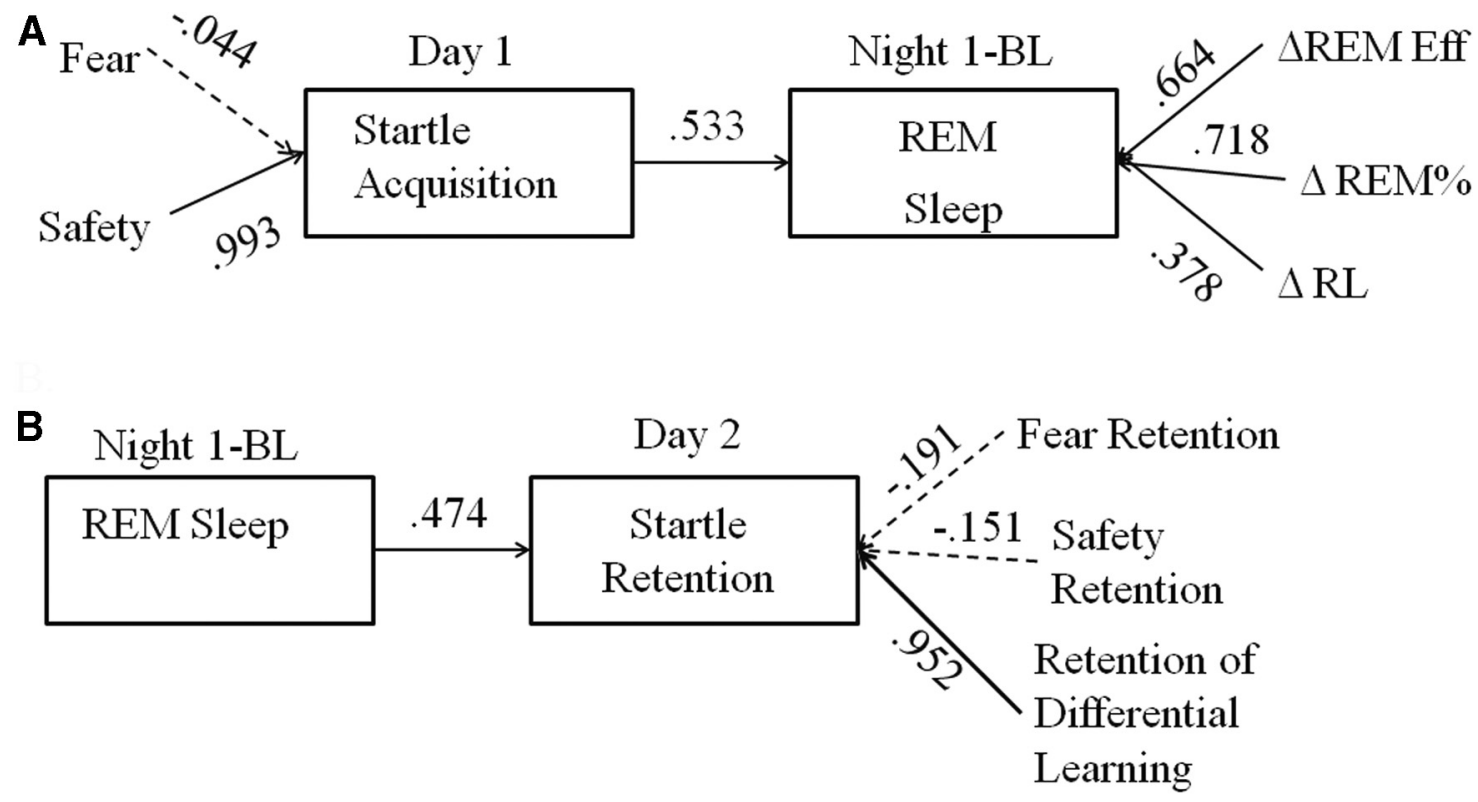

Figure 4. Canonical correlation results. A, Canonical correlation between Startle Acquisition on Day 1 and change in REM sleep on Night 1. B, Canonical correlation between change in REM sleep on Night 1 and Startle Retention on Day 2. For both, canonical variates (i.e., latent variables) are in the boxes. Arrows connecting canonical variates indicate the relationship between the two canonical variates; value is the canonical correlation between the variates. Measures associated with each variate are connected by arrows, and values along the arrows represent the correlations between the measures and the associated canonical variate. Any value $>|0.30|$ is considered to represent a meaningful contribution to the canonical variate structure (solid arrow).

the variance in REM sleep changes from baseline to Night 1 accounted for by safety signal learning. Interestingly, increased REM sleep was, in turn, related to overnight consolidation of the ability to discriminate between the threat and safety stimuli (i.e., differential conditioning). Overall, the REM sleep canonical variate accounted for $22.5 \%$ of the variance in startle retention the next day.

Based on the animal literature, we anticipated that the strength of fear potentiation would predict REM sleep fragmentation, and we did not find that. This may relate to the extent of fear generated by the FPS procedure. The shock we delivered, although aversive, was not as strong or putatively aversive as that delivered in animal studies and certainly not as strong as that experienced by humans during trauma exposure. Alternatively, it may be that sleep in animals is equally sensitive to fear and safety signal learning, and future studies should examine this question across species. Although no other studies report the relationship between safety learning and sleep, animal and human studies show extinction recall and/or generalization are related to REM sleep (Silvestri, 2005; Fu et al., 2007; Spoormaker et al., 2010, 2012). Extinction and safety learning are both forms of fear inhibition (Jovanovic and Norrholm, 2011), so the REM sleep-extinction connection reported previously supports the REM sleep-safety signal link observed in the present study.

The overlap in neural substrates of REM sleep and safety learning provides further support for their connection. REM sleep is characterized by high activity in the limbic system, including hippocampus, and medial prefrontal cortex (mPFC) (Braun et al., 1998; Maquet, 2000). The neural circuits for safety learning are not fully determined, but both hippocampus (Pollak et al., 2008) and mPFC (Jovanovic and Norrholm, 2011) are implicated. Within the hippocampus, safety signal learning results in increased neurogenesis and survival of newborn cells in the dentate gyrus (Pollak et al., 2008). Preventing this neurogenesis slows safety learning significantly, including preventing safety learning in the first day of training. REM sleep promotes neurogenesis, particularly cell proliferation and survival, and REM sleep deprivation impairs those processes (Guzman-Marin et al., 2008; Meerlo et al., 2009). Neurogenesis during REM sleep is important for memory consolidation (Meerlo et al., 2009; Pan et al., 2013), and it may be specifically related to extinction memory (Pan et al., 2013). Although causal relationships were not tested in the present study, this literature suggests that an increase in REM sleep subsequent to safety learning may allow increased neurogenesis, which in turn may contribute to enhanced fear/safety retention and/or discrimination the next day.

The notion that increased REM sleep may help play a role in overnight startle retention receives further support from studies showing that REM sleep plays an active role in consolidation of emotional memories. A growing number of human studies demonstrate that REM sleep plays a facilitatory role in consolidation of emotional memory (Nishida et al., 2009; Baran et al., 2012; Payne et al., 2012; Groch et al., 2013), including positive emotions (Gujar et al., 2011), which are arguably closer to safety signals than the negative emotions typically studied. Several of these studies report that REM sleep increases emotional reactivity, as well as enhancing memory (Gujar et al., 2011; Baran et al., 2012; Groch et al., 2013), helping explain why the ability to discriminate threat from safety showed the most robust association with prior REM sleep. Nishida et al. (2009) further showed that prefrontal theta activity during REM sleep was related to emotional memory consolidation, providing a link to the vmPFC role in safety learning proposed by Jovanovic and Norrholm (2011).

This study implies a causal chain whereby increased safety signal learning during the day influences increased REM sleep consolidation that night, which in turn influences the increased ability to discriminate threat from safety the next day. Two alternative explanations to this chain were considered. First, the FPSREM sleep relationship may be more general and trait-like rather than specific and state-like. That is, perhaps those who more easily adapt to fear and/or more easily learn safety are also those who more easily adapt to a new sleeping environment. This might 
make sense evolutionarily because a new sleeping environment appears to be a stressor in some animals. If the FPS-REM sleep relationship is trait-like, one would expect that the initial change in REM sleep from adaptation night to baseline night would predict initial fear and/or safety acquisition. However, this was not the case, thus arguing that the FPS-REM sleep relationship is state-like and directional. Second, it is possible that initial startle acquisition accounts for both REM sleep and startle recall, so REM sleep, itself, does not account for unique variance in startle recall. An analysis, including both the startle and REM sleep canonical variates as predictors of startle recall, showed that REM sleep does have an independent and significant effect on startle retention beyond that accounted for by initial startle acquisition.

The findings in this study have potential implications for understanding the physiological mechanism underlying the prominent role of sleep difficulties in PTSD. Sleep disruption, especially nightmares, subsequent to trauma exposure predicts the onset of PTSD (Babson and Feldner, 2010; Germain, 2013; van Liempt et al., 2013). One behavioral feature salient in the development and maintenance of PTSD is avoidance, in part because of an inability to accurately discriminate threatening environments from safe environments (Foa and Kozak, 1986). If increased REM sleep consolidation facilitates discriminating threat from safety at the physiological level (via overnight retention of conditioning), the REM sleep disruption characteristic of acute trauma responses and PTSD may impair the ability to retain learning associated with safety and/or threat-safety discrimination. Indeed, impaired safety signal learning appears to be unique to PTSD among clinical populations (Jovanovic et al., 2010), and the ability to modulate fear responses to safety signals is impaired in these patients (Jovanovic et al., 2012). Perhaps a stronger clinical parallel is during the treatment of PTSD. Prolonged exposure is one of two gold standard treatments for PTSD. It involves, among other things, exposure to avoided environments in an effort to extinguish the fear response and allow the patient to relearn the environment is safe. If the REM sleep deprivation produced by nightmares, insomnia, and/or early morning awakenings in PTSD (Spoormaker and Montgomery, 2008; Germain, 2013) impairs the ability to retain learning of threat-safety discrimination and/or the ability to retain newly formed safety memories, such sleep disruptions may reduce the ability to benefit maximally from a treatment, such as prolonged exposure. Although there are studies showing that PTSD treatments do not correct sleep problems (Nappi et al., 2012), none has examined whether nightmares or insomnia during treatment predicts worse outcomes. The clinical connection between sleep and PTSD before, during, and after treatment remains a critical area of study (Germain, 2013).

Although the clinical implications of these findings are important, we must note they were not the focus of study. Rather, we enrolled healthy controls in an effort to take necessary initial steps in advancing translation of relevant animal models into humans. Future research is needed to extend these findings and to test our proposed hypotheses of REM sleep playing a role in the development and/or maintenance of PTSD. One such study would be to actively manipulate REM sleep to test the causal role of REM sleep in fear and safety learning. Spoormaker et al. (2012) reported experimental REM sleep deprivation impaired measures of extinction recall, another process clearly important in PTSD. Those findings further argue for studies systematically testing the effect of manipulating REM sleep on acquisition and/or recall of fear and safety signals. Another avenue for future studies would be to more formally determine whether REM sleep partially or fully mediates the relationship between initial FPS responses on the acquisition day and startle retention. The pattern in our data (startle acquisition response affects subsequent REM sleep, which affects subsequent startle retention) and our post hoc analyses suggests such a meditational role, although we could not formally test that here because it would require $\sim 3$ times as many subjects as we had in this study (Fritz and Mackinnon, 2007). Finally, studies need to test these relationships directly in clinical samples, as well as examine the effects of improving sleep, especially REM sleep, on clinical symptoms and/or treatment response in PTSD.

\section{References}

Acheson D, Feifel D, de Wilde S, McKinney R, Lohr J, Risbrough V (2013) The effect of intranasal oxytocin treatment on conditioned fear extinction and recall in a healthy human sample. Psychopharmacology (Berl) 229: 199-208. CrossRef Medline

Babson KA, Feldner MT (2010) Temporal relations between sleep problems and both traumatic event exposure and PTSD: a critical review of the empirical literature. J Anxiety Disord 24:1-15. CrossRef Medline

Baran B, Pace-Schott EF, Ericson C, Spencer RM (2012) Processing of emotional reactivity and emotional memory over sleep. J Neurosci 32:10351042. CrossRef Medline

Braun AR, Balkin TJ, Wesensten NJ, Gwadry F, Carson RE, Varga M, Baldwin P, Belenky G, Herscovitch P (1998) Dissociated pattern of activity in visual cortices and their projections during human rapid eye movement sleep. Science 279:91-95. CrossRef Medline

Foa EB, Kozak MJ (1986) Emotional processing of fear: exposure to corrective information. Psychol Bull 99:20-35. CrossRef Medline

Fritz MS, Mackinnon DP (2007) Required sample size to detect the mediated effect. Psychol Sci 18:233-239. CrossRef Medline

Fu J, Li P, Ouyang X, Gu C, Song Z, Gao J, Han L, Feng S, Tian S, Hu B (2007) Rapid eye movement sleep deprivation selectively impairs recall of fear extinction in hippocampus-independent tasks in rats. Neuroscience 144: 1186-1192. CrossRef Medline

Germain A (2013) Sleep disturbances as the hallmark of PTSD: where are we now? Am J Psychiatry 170:372-382. CrossRef Medline

Germain A, Buysse DJ, Nofzinger E (2008) Sleep-specific mechanisms underlying posttraumatic stress disorder: integrative review and neurobiological hypotheses. Sleep Med Rev 12:185-195. CrossRef Medline

Grillon C (2002) Startle reactivity and anxiety disorders: aversive conditioning, context, and neurobiology. Biol Psychiatry 52:958-975. CrossRef Medline

Groch S, Wilhelm I, Diekelmann S, Born J (2013) The role of REM sleep in the processing of emotional memories: evidence from behavior and event-related potentials. Neurobiol Learn Mem 99:1-9. CrossRef Medline

Gujar N, McDonald SA, Nishida M, Walker MP (2011) A role for REM sleep in recalibrating the sensitivity of the human brain to specific emotions. Cereb Cortex 21:115-123. CrossRef Medline

Guzman-Marin R, Suntsova N, Bashir T, Nienhuis R, Szymusiak R, McGinty D (2008) Rapid eye movement sleep deprivation contributes to reduction of neurogenesis in the hippocampal dentate gyrus of the adult rat. Sleep 31:167-175. Medline

Johnson LR, McGuire J, Lazarus R, Palmer AA (2012) Pavlovian fear memory circuits and phenotype models of PTSD. Neuropharmacology 62: 638-646. CrossRef Medline

Jovanovic T, Norrholm SD (2011) Neural mechanisms of impaired fear inhibition in posttraumatic stress disorder. Front Behav Neurosci 5:1-8. CrossRef Medline

Jovanovic T, Norrholm SD, Blanding NQ, Davis M, Duncan E, Bradley B, Ressler KJ (2010) Impaired fear inhibition is a biomarker of PTSD but not depression. Depress Anxiety 27:244-251. CrossRef Medline

Jovanovic T, Kazama A, Bachevalier J, Davis M (2012) Impaired safety signal learning may be a biomarker of PTSD. Neuropharmacology 62:695704. CrossRef Medline

Maquet P (2000) Functional neuroimaging of normal human sleep by positron emission tomography. J Sleep Res 9:207-231. CrossRef Medline

Meerlo P, Mistlberger RE, Jacobs BL, Heller HC, McGinty D (2009) New neurons in the adult brain: the role of sleep and consequences of sleep loss. Sleep Med Rev 13:187-194. CrossRef Medline

Milad MR, Goldstein JM, Orr SP, Wedig MM, Klibanski A, Pitman RK, Rauch SL (2006) Fear conditioning and extinction: influence of sex and men- 
strual cycle in healthy humans. Behav Neurosci 120:1196-1203. CrossRef Medline

Nadorff MR, Nazem S, Fiske A (2011) Insomnia symptoms, nightmares, and suicidal ideation in a college student sample. Sleep 34:93-98. Medline

Nappi CM, Drummond SP, Hall JM (2012) Treating nightmares and insomnia in posttraumatic stress disorder: a review of current evidence. Neuropharmacology 62:576-585. CrossRef Medline

Neylan TC, Marmar CR, Metzler TJ, Weiss DS, Zatzick DF, Delucchi KL, Wu RM, Schoenfeld FB (1998) Sleep disturbances in the Vietnam generation: findings from a nationally representative sample of male Vietnam veterans. Am J Psychiatry 155:929-933. Medline

Nishida M, Pearsall J, Buckner RL, Walker MP (2009) REM sleep, prefrontal theta, and the consolidation of human emotional memory. Cereb Cortex 19:1158-1166. CrossRef Medline

Norrholm SD, Jovanovic T, Vervliet B, Myers KM, Davis M, Rothbaum BO, Duncan EJ (2006) Conditioned fear extinction and reinstatement in a human fear-potentiated startle paradigm. Learn Mem 13:681-685. CrossRef Medline

Norrholm SD, Jovanovic T, Olin IW, Sands LA, Karapanou I, Bradley B, Ressler KJ (2011) Fear extinction in traumatized civilians with posttraumatic stress disorder: relation to symptom severity. Biol Psychiatry 69: 556-563. CrossRef Medline

Ohayon MM, Shapiro CM (2000) Sleep disturbances and psychiatric disorders associated with posttraumatic stress disorder in the general population. Compr psychiatry 41:469-478. CrossRef Medline

Pace-Schott EF, Milad MR, Orr SP, Rauch SL, Stickgold R, Pitman RK (2009) Sleep promotes generalization of extinction of conditioned fear. Sleep 32:19-26. Medline

Pan YW, Storm DR, Xia Z (2013) Role of adult neurogenesis in hippocampus-dependent memory, contextual fear extinction and remote contextual memory: new insights from ERK5 MAP kinase. Neurobiol Learn Mem 105:81-92. CrossRef Medline

Payne JD, Chambers AM, Kensinger EA (2012) Sleep promotes lasting changes in selective memory for emotional scenes. Front Integr Neurosci 6:108. CrossRef Medline

Pollak DD, Monje FJ, Zuckerman L, Denny CA, Drew MR, Kandel ER (2008) An animal model of a behavioral intervention for depression. Neuron 60:149-161. CrossRef Medline

Polta SA, Fenzl T, Jakubcakova V, Kimura M, Yassouridis A, Wotjak CT (2013) Prognostic and symptomatic aspects of rapid eye movement sleep in a mouse model of posttraumatic stress disorder. Front Behav Neurosci 7:60. CrossRef Medline

Sanford LD, Silvestri AJ, Ross RJ, Morrison AR (2001) Influence of fear conditioning on elicited ponto-geniculo-occipital waves and rapid eye movement sleep. Arch Ital Biol 139:169-183. Medline

Sanford LD, Fang J, Tang X (2003) Sleep after differing amounts of conditioned fear training in BALB/cJ mice. Behav Brain Res 147:193-202. CrossRef Medline

Silvestri AJ (2005) REM sleep deprivation affects extinction of cued but not contextual fear conditioning. Physiol Behav 84:343-349. CrossRef Medline

Spoormaker VI, Montgomery P (2008) Disturbed sleep in post-traumatic stress disorder: secondary symptom or core feature? Sleep Med Rev 12: 169-184. CrossRef Medline

Spoormaker VI, Sturm A, Andrade KC, Schröter MS, Goya-Maldonado R, Holsboer F, Wetter TC, Sämann PG, Czisch M (2010) The neural correlates and temporal sequence of the relationship between shock exposure, disturbed sleep and impaired consolidation of fear extinction. J Psychiatr Res 44:1121-1128. CrossRef Medline

Spoormaker VI, Schröter MS, Andrade KC, Dresler M, Kiem SA, GoyaMaldonado R, Wetter TC, Holsboer F, Sämann PG, Czisch M (2012) Effects of rapid eye movement sleep deprivation on fear extinction recall and prediction error signaling. Hum Brain Mapp 33:2362-2376. CrossRef Medline

Sturm A, Czisch M, Spoormaker VI (2013) Effects of unconditioned stimulus intensity and fear extinction on subsequent sleep architecture in an afternoon nap. J Sleep Res 22:648-655. CrossRef Medline

Tabachnick BG, Fidell LS (2001) Canonical correlation. In: Using multivariate statistics, pp 177-218. Boston: Allyn and Bacon.

Thompson B (2000) Canonical correlation analysis. In: Reading and understanding more multivariate statistics (Grimm LG, Yarnold PR, eds), pp 285-316. Washington, DC: American Psychological Association.

van Liempt S, van Zuiden M, Westenberg H, Super A, Vermetten E (2013) Impact of impaired sleep on the development of PTSD symptoms in combat veterans: a prospective longitudinal cohort study. Depress Anxiety 30:469-474. CrossRef Medline

Wellman LL, Yang L, Tang X, Sanford LD (2008) Contextual fear extinction ameliorates sleep disturbances found following fear conditioning in rats. Sleep 31:1035-1042. Medline 